\title{
Survey Builder: A Tool to Support Assessment
}

\author{
Marc Hoit ${ }^{1}$, Rick Sayers ${ }^{2}$, Bill Lewis ${ }^{2}$, Akhil Karkera ${ }^{2}$, Naren Kamat ${ }^{2}$ \\ ${ }^{1}$-Associate Dean, College of Engineering, Academic Affairs/ \\ ${ }^{2}$-Career Resource Center \\ Univer sity of Florida, Gainesville, FL
}

Assessment is a critical component of all educational programs. The need to develop and administer surveys to a wide variety of audiences is one of the standard techniques used in all assessment programs. One of the major difficulties in this assessment technique is the administering, collecting and reducing of the resulting data. This difficulty becomes more pronounced as data is collected across many years and different constituent groups. In order to improve the collection and reduction of this type of data, the Career Resource Center (CRC) has partnered with the College of Engineering in order to develop a dynamic web based survey tool. The Survey Builder application designed for the University of Florida is a tool that lets users create surveys, administer them, and analyze survey results all through the web.

The Survey Builder is a general tool that has been implemented across the entire campus. It allows any department to create a survey and administer it over the web. Questions can be Likert scale, yes/no, short answer or open response. Each question has a unique ID and can be re-used in subsequent surveys. Colleges have the ability to develop standard questions that are included in all departmental surveys. All results are stored in a database accessible by the department that creates the survey.

The Survey Builder has been tested in two departments during the fall 2003 semester for undergraduate exit surveys. Spring 2004, the tool was extended to include all engineering departments in the college. The results of these tests and the effectiveness of the tool will be discussed.

\section{Motivation for Development}

In addition to needing survey information as one component of the ABET process, the college and university wish to keep track of additional information about our students. The following is an example of some of the additional information we hope to track:

- Determine the post graduation destinations of students

- Determine the comparative success of UF students to national norms 
- Determine who is and who is not recruiting UF students

- Gather career advising information which will help inform student of career choices

- Be able to track career progress of Alumni

In the past, many of our departments have used paper based collection systems for the ABET student exit interview, and alumni survey. This puts an additional burden of transferring the paper survey into a form that can be tracked and compared. Some of our departments started developing single use survey systems. Engineering decided to build a comprehensive web-based surveying tool in partnership with the Career Resource Center. This allowed colleges and/or departments on campus to standardize the gathering of statistical and qualitative information needed for accreditation and other tracking issues. The "Destinations" survey building tool and exit survey process is our solution to creating a universal system.

\section{Description of Survey Tool "Destinations"}

The survey tool has the following capabilities and features:

- Ability to allow for the creation of any number of surveys and associate them to a University entity (College, Department, Division, etc.)

- Designate questions by the college that are used in all department surveys

- Ability to create many different types of survey questions (radio buttons, check boxes, free form text fields, etc.)

- Ability to add sections to help group questions within the surveys

- Ability to allow for secure transactions for students entering data (SSL)

- Integration into the GatorLink (campus) authentication system

- Renumbering of the questions and the sections is done automatically upon reordering either the sections or the questions, thus maintaining consistency

- Preview mode available to correct errors and make modifications

- Mandatory fields can be set as required, and removed if not needed

- Permission-based access to the administrative area

- Survey results are stored in a database with unique IDs for questions

- A statistics reporting tool in included that displays graphs and percentage values by categorization

- An Excel spreadsheet report that shows the responses of all the students to each of the questions can be downloaded.

- The survey tool has an incorporated timeout mechanism that forces the users to login again if there is a period of inactivity on the screen. This protects against some other user taking advantage of a logged-in session.

- The survey system tool does not allow a student to respond to the survey twice. If a student has already taken the survey, it will not allow that student to resubmit the survey again under the same student-id.

- The tool gives the departments the option to indicate whether they want the surveys to be conducted anonymously. If so indicated at the time of creation, no demographic information is requested of the respondents at the start of the survey. 
- Even if demographic information is collected, the reporting tool does not give any option to link the answers to their corresponding respondents unless explicitly requested by the department. As such, the privacy of the respondents is ensured according to the department policy.

\section{Broad Description of System Features}

The development of the survey system tool was done using the Microsoft SQL Server 2000 database as the backend. We used Active Server Pages technology to develop the web-based interface for the project. The following is a list of the basic functions of the survey system;

1. Create Survey: The system allows the authorized user to create a survey. At his/her disposal the user has several question types to choose from. They can group questions in to sections for better understanding.

2. Administer Survey: Once the survey is complete, the system will let the user specify email addresses of students that the survey link is emailed to.

3. Take Survey: Students can $\log$ in to the system using their University of Florida student id numbers to take the survey.

4. View survey results: Once the survey is complete, the authorized user can view survey results. The results can be downloaded as an MS Excel spreadsheet or an MS Access database. They can also access some generic reports that are automatically/dynamically generated for every survey.

5. Linking Surveys: For the benefit of the colleges within the university, college surveys can be linked to department surveys so that each time a student takes a survey created by his department, the college survey is also displayed. This allows colleges to get feedback from their students.

\section{Table Descriptions:}

The following is a list of the internal data tables used to track the resulting survey information;

1. SurveyList: This table keeps records on every survey created by the system. It stores parameters that are applicable to the survey as a whole, such as survey creator, if it is published, the activation dates etc... It also stores the college survey and the CRC survey reference number for surveys that are associated with the department survey.

2. SurveyListLog: This table holds surveys that have been deleted. This is for accounting purposes, so that old records from the answers table can be associated with a survey even if it has been deleted.

3. Department Survey tables: Every department has four primary tables and three log tables associated with it. This set of seven tables is replicated for each entity in the survey builder system. So, each department gets its own set of seven tables. These tables are differentiated from each other on the basis of the three letter department code that precedes each table name. For example, the Material Science and Engineering department (MSE) has the following tables; 
a. MSESections: This table holds section details for a section of the survey. There can be multiple sections for a single survey, hence the one to many relationship

b. MSEQuestions: This table holds question details for a question of the survey. Again, there can be multiple questions for a single survey, hence the one to many relationship

c. MSEAnswer Choices: This table holds answer choices for the custom radio box and he custom check box types of questions

d. MSEAnswers: This table holds answers that the student has entered for the survey. The design of this table is such that every answer is stored as a text field, and is then interpreted depending on the kind of question that the answer is associated with. This design allows for different question types to be added easily into the system.

e. MSEQuestionsLog, MSEAnswer sChoicesLog, MSESectionsLog: These tables store deleted questions. The deleted questions still have the old question reference number. This process of storing deleted questions, allows for survey versioning. Hence when viewing survey results we can map answers to different questions over a period of time.

4. College Survey tables: Like the department every college has four primary tables and three $\log$ tables associated with it. The college tables are again differentiated from each other and the department tables on the basis of the three letter college code that precedes each table name. The functions of the respective tables are the same as the department tables.

5. CRC Survey tables: These again follow the pattern of the department and college tables.

6. Usernames: The usernames table stores user details. We don't store user passwords as this is taken care of by the Gatorlink campus-wide ID system. Each time a user logs in, he is authenticated against the Gatorlink system, and only the username is passed on. The usernames table has details about which entity the user belongs to. This helps to load the appropriate department/college view when the user logs in

7. Permissions: Each user has permissions assigned to perform the various tasks in the system. Some examples of tasks are: "Read", "Modify", "Administer" and "View Results". The permissions table maps the username to the task

8. TaskTypes: This table shows the various different tasks that the user can perform within the system. If a need arises more task types can be added to this list. This list is used by the permissions table when the username is first assigned a task type.

9. RoleTypes: The Permissions table can optionally have a role assigned to a username. This allows the administrator to group several tasks into one role and simply assign that role to the user, thereby assigning corresponding tasks to him too. For instance an "OnlyView" role can include the "Read" and "View Results" tasks within it.

10. SurveyTypes: This table stores the different types of surveys. When creating a survey the department user can give it a type name (populated from this list). The College user can then associate a particular college survey with all department surveys that match that survey type. From then on, all department surveys that are created with that survey type will have the college survey automatically linked to their survey. This eases the burden on the college user to keep rescanning department surveys to find surveys that can be linked with his college survey.

11. QuestionTypes: This is a simple list of different types of questions. This table populates the question type menu when creating a question.

"Proceedings of the 2004 American Society for Engineering Education Annual Conference \& Exposition Copyright (C) 2004, American Society for Engineering Education” 


\section{Example Screen Shots}

The following are example screen shots of the key system functions. First is what the person taking the survey sees. The following three screens are the user login and an example of the survey forms that a participant will see. All screens are automatically generated from the survey defined by the department survey administrator.

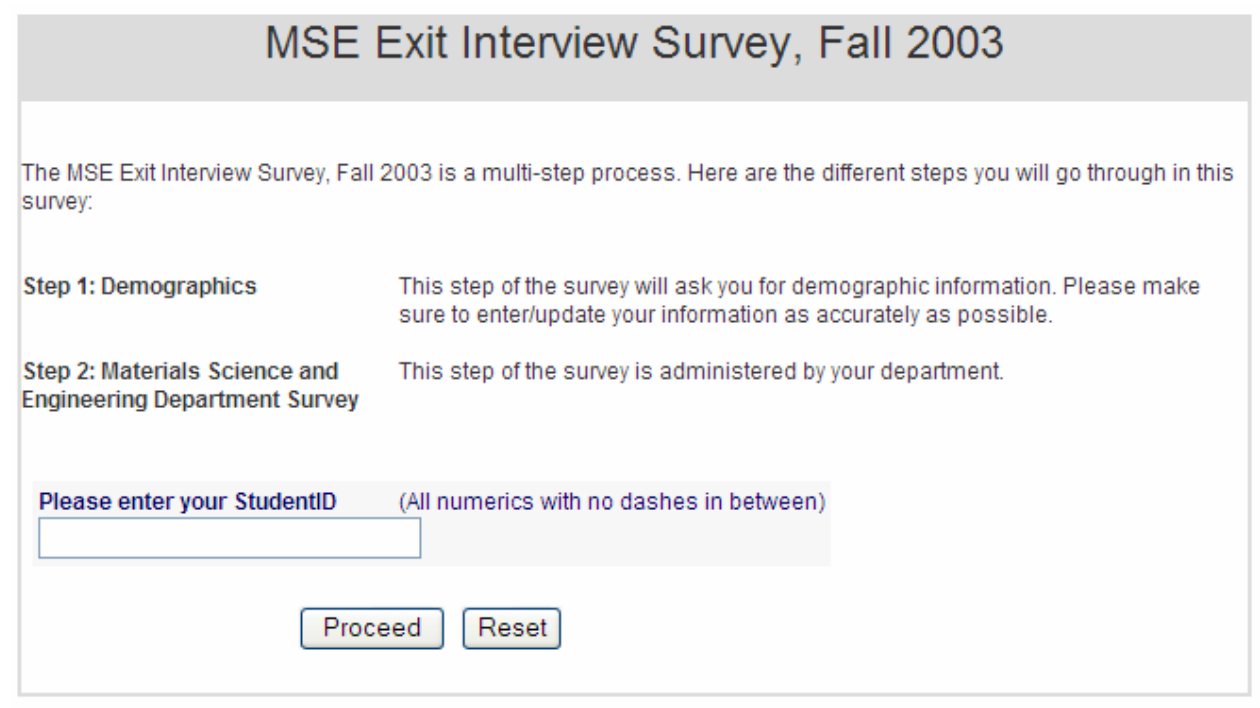

Fig 1. Initial student login screen.

This is the user login-in screen. It requires the student to enter a secure ID in order to access their unique data. 
Session 2158

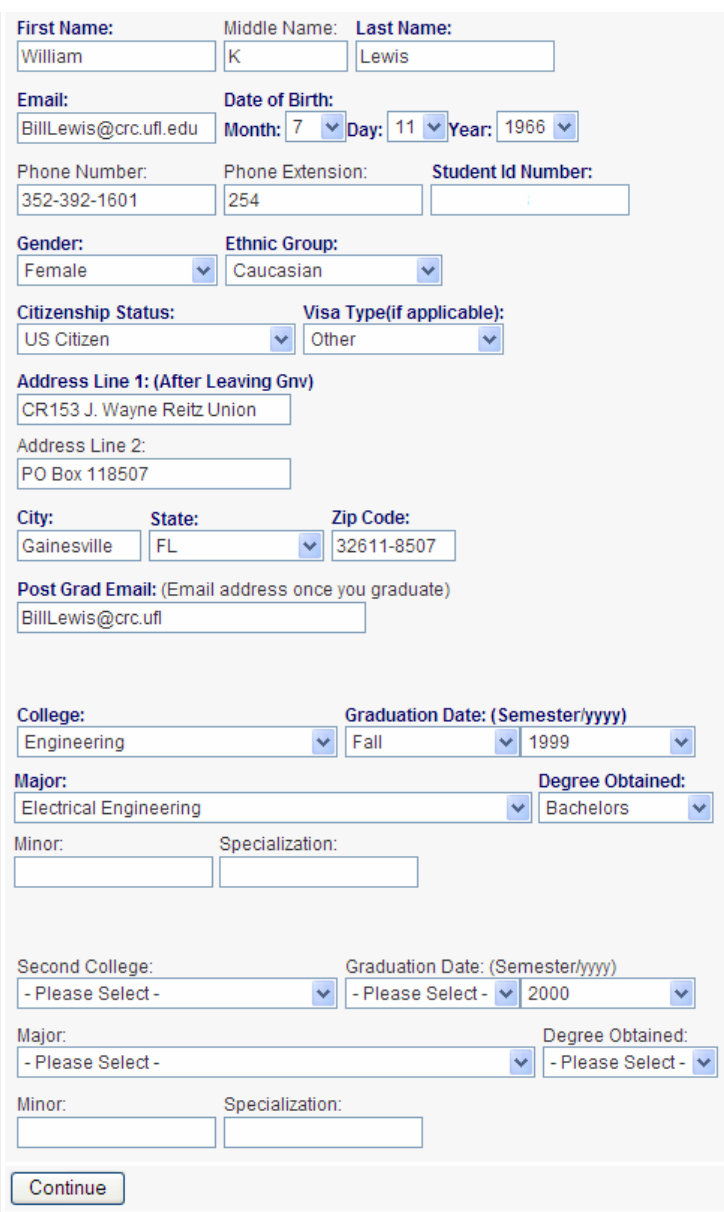

Fig 2. Student Demographics screen.

Next is the demographics screen. This section collects the student demographic information. If the student has previously used the services of the Career Resource Center, this screen will be automatically filled from the student records database. It allows the student to update their records for tracking purposes. Since this is not a university-wide record, and is created by the students themselves, either during this survey or while using some other online service available through the Career Resource Center, it allows the students to modify their record, if they wish to indicate any changes in it. 


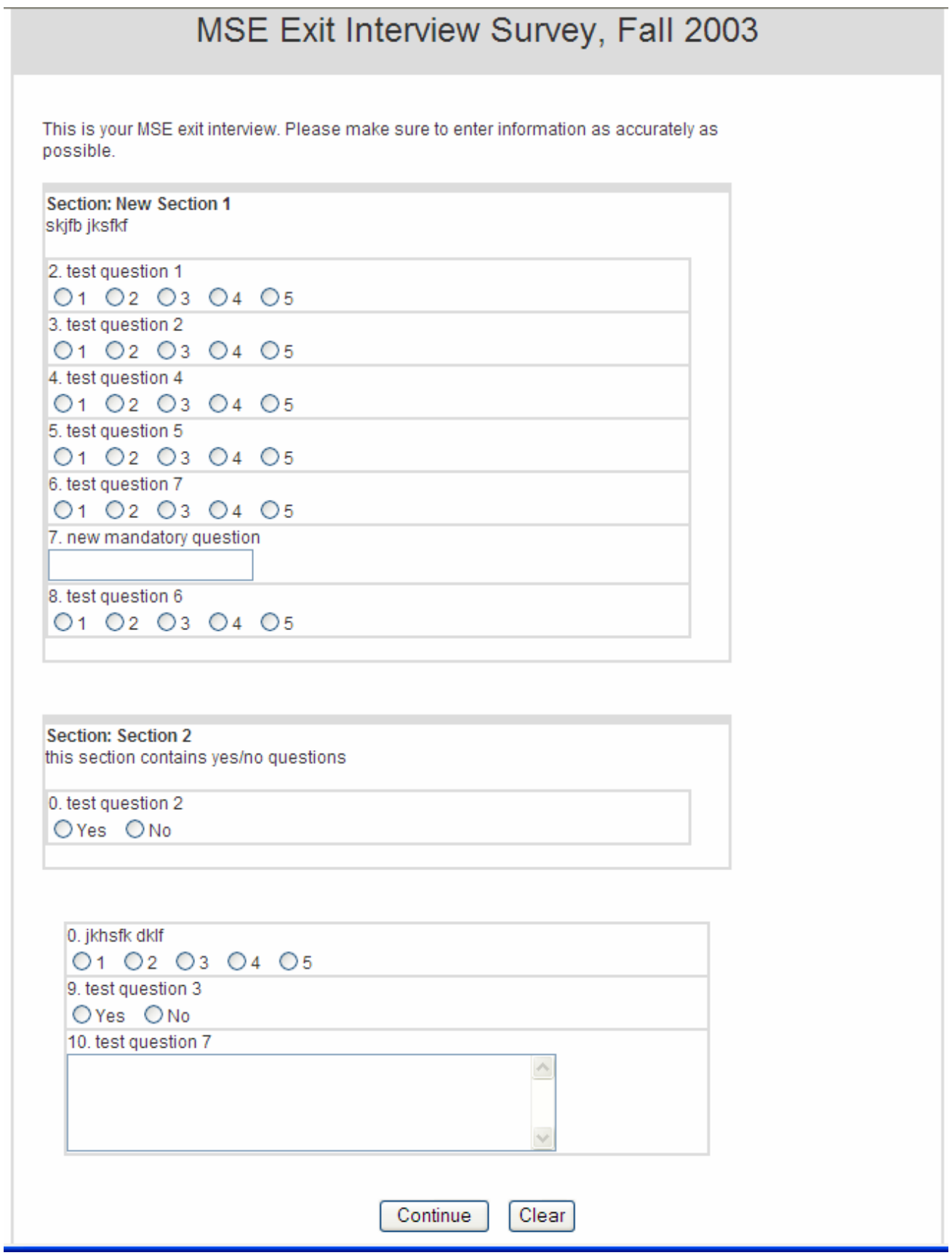

Fig 3. Survey Questions screen

Finally, the student will see the actual survey questionnaire.

Next are the administrator section screens. There is an administrator defined for each department as well as a college administrator. The college administrator can define questions that are used in all the departmental surveys. This allows for common questions across disciplines. The departmental administrator can define and develop all new surveys. 
Session 2158

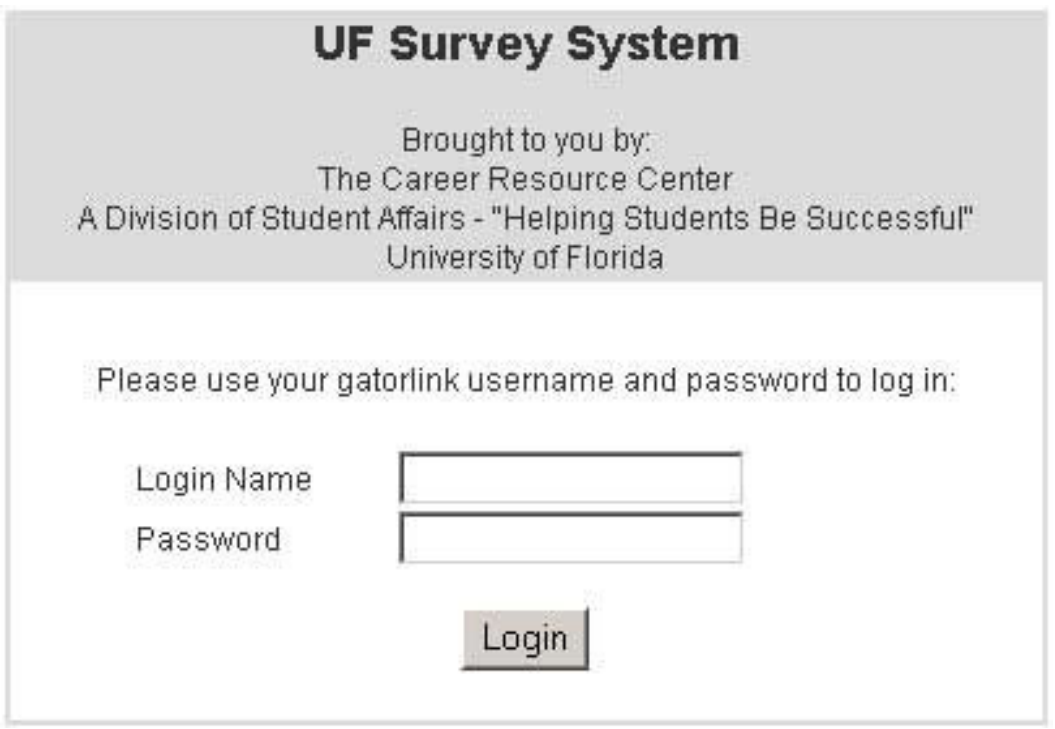

Fig 4. Survey administrator login screen.

This is the initial login screen that the administrators have to go through. The authentication mechanism uses the secure campus-wide "Gatorlink" authentication mechanism. It authenticates the users by using their university-assigned and managed usernames and passwords. At the time of initial setup, each department is required to submit a list of authorized people along with their corresponding "Gatorlink" usernames. Only these authorized people are able to login to the system using this screen.

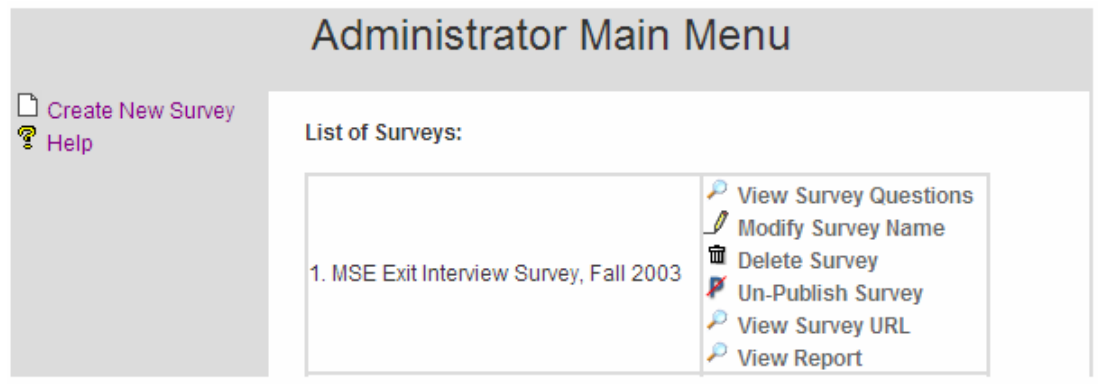

Fig 5. Survey administrator menu after authentication.

This screen shows the current surveys available to the Materials Science Department. Currently there is only one survey available. From this screen, the department can create new surveys. Once a survey has been published, it cannot be changed unless it is unpublished again. 
Session 2158

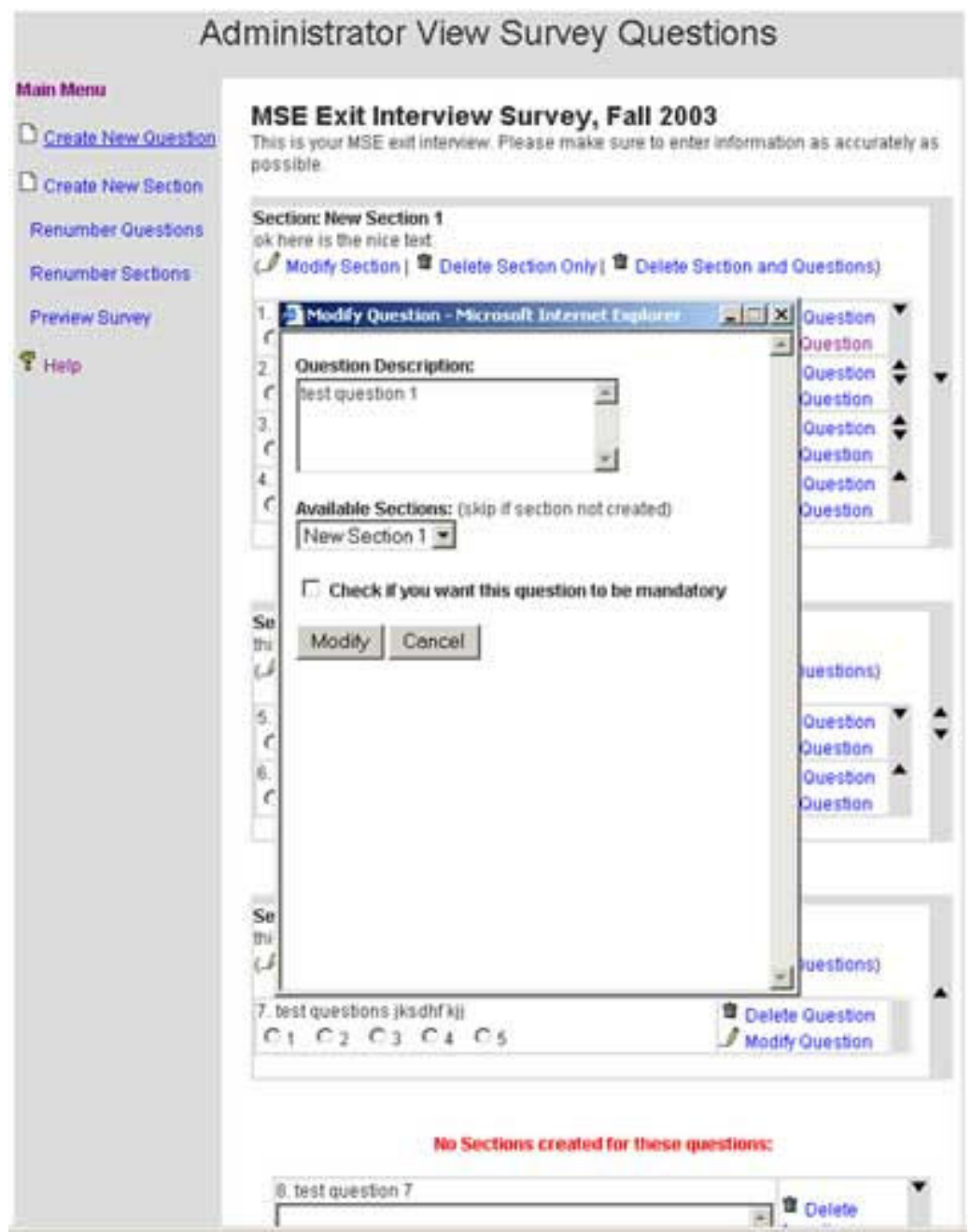

Fig 6. Sample administrative tool functionality.

This final screen shows how questions and sections are created by the administrator. Note how the sections help organize the questions into logical groups. The up and down arrows on the right allow the user to renumber the questions within the sections. The up and down arrows on the outside allow the user to reorganize entire sections. Upon reorganizing, the question numbers are automatically renumbered. 


\section{Report Generation}

The survey tool has the ability to generate graphic reports for specific questions of any survey. It also allows the department to download files in Microsoft Access and/or Excel format with the results of a survey. Files can be downloaded at any time. An example of the graphic display from some questions is given below;

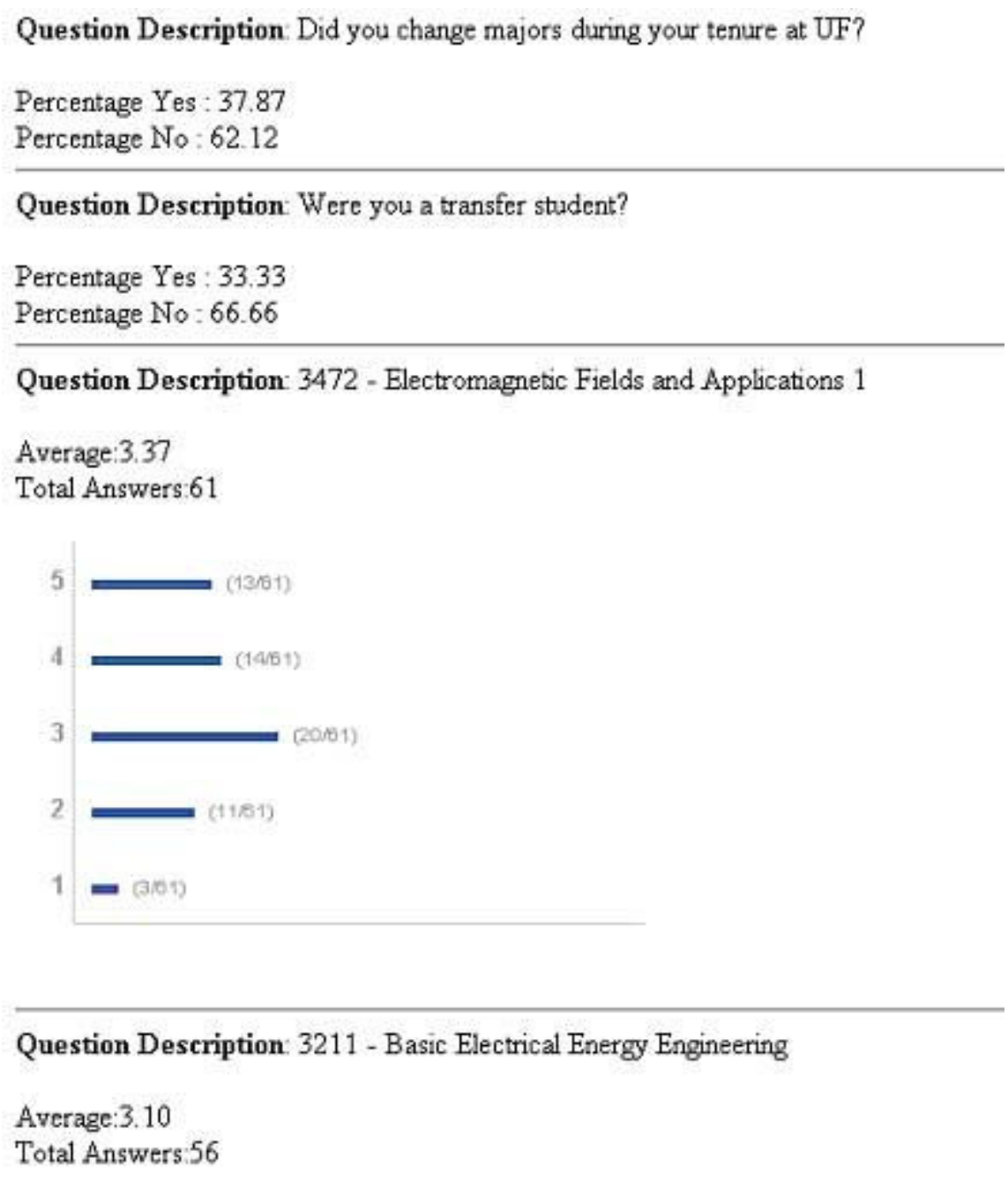

Fig 7. Survey system reporting tool output

Conclusions

The "Destinations" survey system was completed and tested during the Fall 03 semester in two engineering departments. It was used for exit surveys for graduating students. As a result of the pilot testing, we began using the system for all engineering departments and undergraduate business programs during the Spring 04 semester. It will be rolled out to the entire university in fall of 2004. 
The system has been used for exit surveys and several special purpose surveys to this point. It was designed to be used for a much broader set of questionnaires including class assessments, alumni and employer surveys and more.

The system is robust, scalable and flexible. Additional enhancements are planned including:

- As the surveys get modified, the tool will be able provide related reporting with reference to older surveys made on the system.

- Administrative menus for adding colleges and departments within colleges.

- Administrative menus to control the survey creators and the permissions allotted to them.

The two most innovative components of the system is the ability to create JavaScript on the fly in order to validate questions while they are being answered. When an administrator is creating a survey question, they have the ability to make the question "mandatory". By checking this box the system auto generates checking code for the submission process. If a student does not complete a "mandatory" question, they are prompted with a text box that tells them they have missed a mandatory field and are instructed to return to the screen and fill out any field that is noted with a color change. The second innovative component is the ability to create "Custom" checkbox and/or radio button type questions. During testing, the users requested more flexible options for the answer types. Hence, the ability to create custom Likert and radio responses was added. These have proven to be very flexible and the departmental administrators appreciate the enhancement.

The most difficult part of this project was not having the ability to access record data directly from the University records. Allowing the student to self report can be a positive, as students don't necessarily maintain their records after graduation. This self reporting also allows for the student to give us future contact information that may be useful in obtaining additional survey responses after graduation that may not be part of the official information on file with the University.

Overall, the system has been a success and will continue to be enhanced as more departments take advantage of its availability. 